\title{
Electronic-momentum distribution in deformed sodium clusters
}

\author{
A. Rigo, M. Casas, and F. Garcias \\ Departament de Física, Universitat de les Illes Balears, E-07071 Palma de Mallorca, Spain \\ E. Moya de Guerra and P. Sarriguren \\ Instituto de Estructura de la Materia, CSIC, Serrano-119, E-28006 Madrid, Spain
}

(Received 11 December 1997; revised manuscript received 3 February 1998)

\begin{abstract}
We present results for electronic momentum distribution $n(\mathbf{k})$ of deformed sodium clusters in the framework of the Kohn-Sham formalism. We discuss properties of both spheroidal clusters and fission configurations. For spheroidal clusters we have analyzed properties of total and single-particle electronic momentum distribution. The results show that deformation has more of an effect on the latter, and that the total quadrupole moment in $k$ space can be used to search the minimum-energy configuration. With regard to fission configurations, we present the behavior of total quadrupole moment for different fission pathways. [S0163-1829(98)07619-X]
\end{abstract}

Since the discovery of electronic shell structure in free alkali clusters by Knight et al., ${ }^{1,2}$ several models have been applied to understand the static and dynamic properties of metal clusters. In particular, approaches based on the energy density functional theory ${ }^{3,4}$ have provided the one-body density in $r$ space, $n(\mathbf{r})$, for both spherical and deformed clusters, and have given reasonable predictions of many properties such as experimental ionization potentials, ${ }^{5}$ dipole polarizabilities, ${ }^{6}$ and critical numbers for fission of charged clusters. $^{7}$

More recently, predictions of the local momentum distribution ${ }^{8}$ and of the global one-body density in $k$ space, $n(\mathbf{k})$, have received some theoretical attention. ${ }^{9}$ In fact, the question of how electrons move inside clusters can give some information about the minimum cluster size to reach the results of the Fermi gas model for the bulk metal.

The main features of $n(\mathbf{k})$ for spherical sodium clusters have been recently analyzed ${ }^{9}$ using the Kohn-Sham (KS) formalism within the local-density approximation (LDA) for the valence electrons and the jellium approach for the ionic background, and the standard distribution of the Fermi gas model is found to be unrealistic for a cluster size smaller than $\sim 40000$ atoms.

As discussed by de Heer, ${ }^{3}$ there is clear experimental evidence that clusters are deformed in regions between major spherical-shell closures. The experimental mass spectra of these deformed clusters were first explained, to the best of our knowledge, by Clemenger, ${ }^{10}$ and later by Reimann, Brack, and Hansen, ${ }^{11}$ using a modified Nilsson Hamiltonian.

Ekardt and Penzar ${ }^{12,13}$ and Penzar and Ekardt ${ }^{14}$ have confirmed the existence of axially deformed equilibrium shapes in metal clusters within the self-consistent KS spheroidal calculations and the jellium model. Deformation effects can be seen in the ionization potentials, electron affinities and collective photoabsortion spectra. The photoabsortion cross sections for free singly charged sodium clusters (14-60 atoms) have been recently measured, ${ }^{15,16}$ and a double structure of the resonance peak has been observed for nonmagic clusters as a clear signature of the deformation of these clusters. ${ }^{17}$ But there is no indication of how the jellium deformation affects the electronic momentum distribution. The purpose of this paper is to investigate several features of the momentum distribution in deformed clusters and fission configurations ${ }^{18,19}$ using the KS formalism.

We assume that the main properties of metal clusters can be understood from the quantized motion of the valence electrons in the mean field created by their mutual interactions and the positive background described in the jellium model. ${ }^{4}$ The electronic density $n(\mathbf{r})$ has been obtained from the selfconsistent solution of the KS equations,

$$
\left[-\frac{1}{2} \nabla^{2}+\int \frac{d \mathbf{r}^{\prime} n\left(\mathbf{r}^{\prime}\right)}{\left|\mathbf{r}-\mathbf{r}^{\prime}\right|}+V_{\mathrm{xc}}(n(\mathbf{r}))+v_{\mathrm{je}}(\mathbf{r})\right] \varphi_{j}(\mathbf{r})=\epsilon_{j} \varphi_{j}(\mathbf{r}),
$$

where $V_{\mathrm{xc}}(n(\mathbf{r}))$ is the usual exchange-correlation potential within the LDA (for simplicity the Wigner approximation has been used for the correlation part), and $v_{\mathrm{je}}$ is the potential of the ionic background modeled in the jellium approximation (Hartree atomic units have been used throughout the text).

As in Ref. 14, for spheroidal clusters $v_{\text {je }}$ is obtained using an axially symmetric jellium profile with constant density and semiaxes $\rho_{0}$ and $R_{z}$ defined by

$$
R_{z}=\left(\frac{2+\delta}{2-\delta}\right)^{2 / 3} R_{0}, \quad \rho_{0}=\left(\frac{2-\delta}{2+\delta}\right)^{1 / 3} R_{0}
$$

where $R_{0}$ is the radius of the sphere with the same volume, and $\delta$ is restricted by $-2<\delta<2$. The $\mathrm{KS}$ equations are solved in cylindrical coordinates using a two-dimensional evenly spaced finite difference grid. The ground state is determined by solving Eq. (1) for fixed values of the $\delta$ parameter, which is then adjusted to minimize the total energy of the system.

The fission pathway is obtained by selecting an appropriate sequence of jellium configurations. ${ }^{18,19}$ The jellium profile is modeled by two spheres of radius $R_{1}$ and $R_{2}$ joined by a quadratic surface of revolution. ${ }^{20}$ The jellium shape is then determined by the asymmetry parameter $\Delta$ 


$$
\Delta=\frac{R_{1}-R_{2}}{R_{1}+R_{2}},
$$

the distance parameter $\sigma$, which is proportional to the distance $s$ between the centers of the spheres,

$$
\sigma=\frac{s}{R_{1}+R_{2}}
$$

and the neck deformation parameter $\lambda$, proportional to the thickness of the neck (see Ref. 20 for details)

$$
\lambda=\frac{l_{1}+l_{2}}{R_{1}+R_{2}} .
$$

The self-consistent solution of Eq. (1) for each jellium configuration, using the same numerical method described before, provides us with the single-particle wave functions $\left\{\varphi_{j}(\rho, z)\right\}$ and the corresponding eigenenergies $\left\{\epsilon_{j}\right\}$.

For a cluster with $N$ valence electrons we define the density in momentum space analogously to that in real $r$-space computed in cylindrical coordinates

$$
\begin{gathered}
n(\rho, z)=\sum_{j=1}^{N}\left|\varphi_{j}(\rho, z)\right|^{2}, \\
n\left(k_{\rho}, k_{z}\right)=\sum_{j=1}^{N}\left|\widetilde{\varphi}_{j}\left(k_{\rho}, k_{z}\right)\right|^{2},
\end{gathered}
$$

where $\widetilde{\varphi}_{j}\left(k_{\rho}, k_{z}\right)$ is the Fourier transform of the singleparticle wave function.

We also define the multipole components of the densities in an analogous way in $k$ and $r$ space,

$$
\begin{gathered}
n(\rho, z)=n\left(r \cos \theta_{r}, r \sin \theta_{r}\right)=\sum_{j} n_{j}(r) P_{j}\left(\cos \theta_{r}\right), \\
n\left(k_{\rho}, k_{z}\right)=n\left(k \cos \theta_{k}, k \sin \theta_{k}\right)=\sum_{j} n_{j}(k) P_{j}\left(\cos \theta_{k}\right),
\end{gathered}
$$

which allow one to obtain a $j$-polar momentum in both spaces:

$$
\begin{aligned}
Q_{r}^{j} & =\frac{1}{N}\left(\frac{4 \pi}{2 j+1}\right)^{1 / 2} \int_{0}^{\infty} d r r^{j+2} n_{j}(r), \\
Q_{k}^{j} & =\frac{1}{N}\left(\frac{4 \pi}{2 j+1}\right)^{1 / 2} \int_{0}^{\infty} d k k^{j+2} n_{j}(k) .
\end{aligned}
$$

Using the jellium parametrization (2), we have analyzed the neutral $\mathrm{Na}$ deformed clusters with 10, 22, 24, and 42 atoms, and compared the corresponding results with the predictions obtained for magic clusters $\mathrm{Na}_{8}, \mathrm{Na}_{20}$, and $\mathrm{Na}_{40}$, respectively. In agreement with the results by Clemenger, ${ }^{10}$ the deformation parameter $\delta$ and the quadrupole moment in $r$ space [Eq. (10)] show that $\mathrm{Na}_{10}, \mathrm{Na}_{22}, \mathrm{Na}_{24}$, and $\mathrm{Na}_{42}$ are clearly deformed as one can see in Table I. In $k$ space the electronic quadrupole moment (11) is null for the jellium
TABLE I. Deformation parameter and quadrupole moment in $r$ space corresponding to the minimum-energy configuration.

\begin{tabular}{ccc}
\hline \hline & $\delta$ & $Q_{r}^{2}$ (a.u.) \\
\hline $\mathrm{Na}_{10}$ & 0.484 & 11.976 \\
$\mathrm{Na}_{22}$ & 0.217 & 7.407 \\
$\mathrm{Na}_{24}$ & 0.319 & 12.124 \\
$\mathrm{Na}_{42}$ & 0.394 & 22.765 \\
\hline \hline
\end{tabular}

configuration that corresponds to the minimum energy (see Fig. 1); this can be used to search the value of the $\delta$ parameter for the ground state.

This property can be shown analytically for an anisotropic $\mathrm{HO}$ and follows from the condition that the shapes of the density and the potential be equal. ${ }^{21,22}$ However, as in the nuclear physics case, ${ }^{23}$ in deformed KS calculations, a priori, there is no guarantee that this property be satisfied. We find here that our KS results have this property for each equilibrium deformation obtained by minimization of the energy.

It is worth mentioning that in spite of the null value for the quadrupole moment in $k$ space, the global momentum distribution shows a small anisotropy that comes from the oscillations of the quadrupole component $n_{2}(k)$, which averaged over $k^{4}(11)$ gives a null value of $Q_{k}^{2}$.

The main properties of the global electronic momentum distribution can be understood from its monopole component $n_{0}(k)$. We have verified that, in analogy to what happens with the monopole density in $r$ space, shell effects on $n_{0}(k)$ diminish with deformation. The surface thickness in $r$ space increases with deformation and, consequently, $n_{0}(k)$ for small $k$ values increases. This feature has been observed in the momentum distribution of spherical clusters ${ }^{9}$ and can be explained on the basis of the Slater approach for the local momentum distribution.

We have also studied the effect of deformation on the single-particle momentum distribution. As long as the system loses its spherical symmetry, the angular momentum quantum number $l$ is no longer a good quantum number. Therefore, in the single-particle orbitals corresponding to a deformed system we found a mixture of different $l$ components. We have verified that there is no general rule for ana-

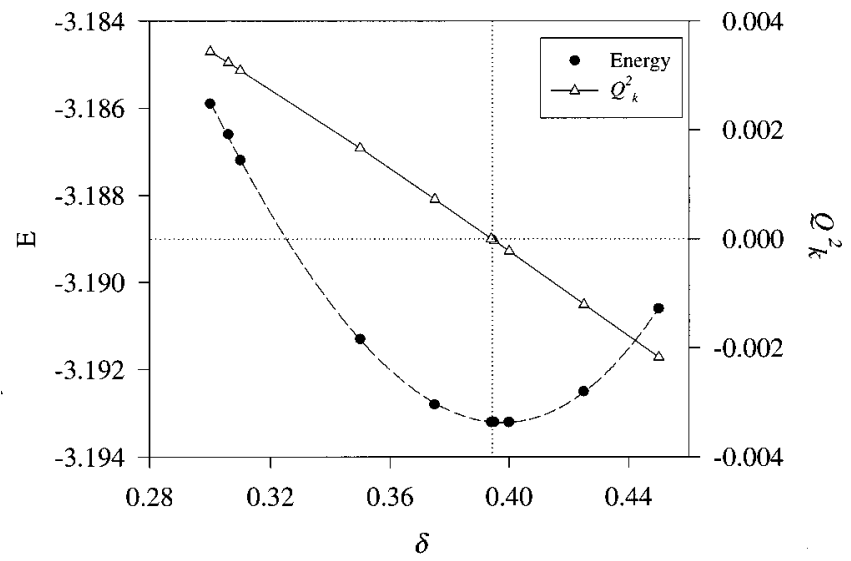

FIG. 1. Energy and quadrupole moment in $k$ space vs $\delta$ for $\mathrm{Na}_{42}$ in Hartree atomic units. 


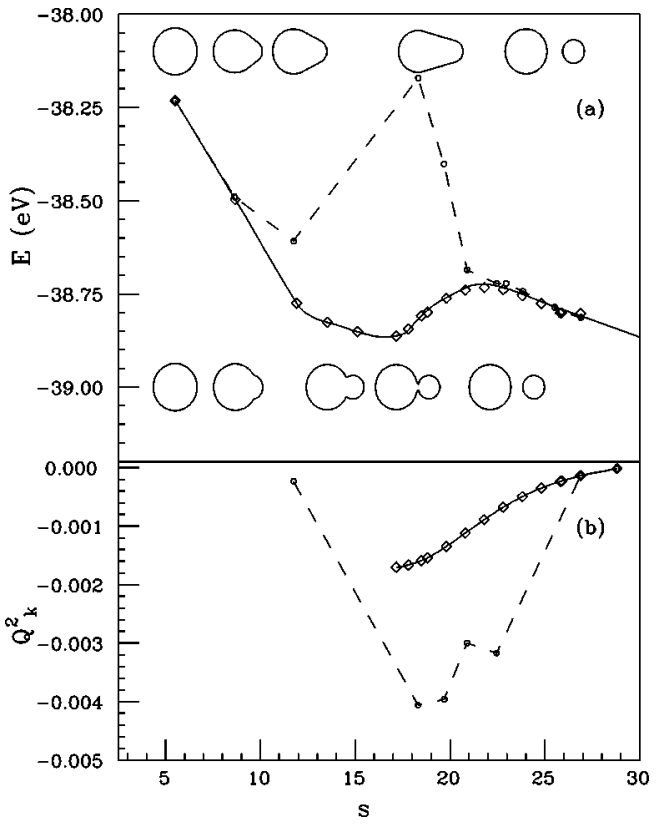

FIG. 2. Different fission pathways for the process $\mathrm{Na}_{24}^{2+}$ $\rightarrow \mathrm{Na}_{21}^{+}+\mathrm{Na}_{3}^{+}$(a). The $Q_{k}^{2}$ values in Hartree atomic units for each jellium configuration are also shown (b).

lyzing the deformation effects in each single-particle orbital, but the differences on the single-particle momentum distributions between spherical and deformed orbitals can be seen as a signature of the mixtures of different $l$ components.

The global momentum distribution for different fission pathways has been also analyzed using appropriate jellium configurations. We have considered the charged trimer emission from $\mathrm{Na}_{24}^{2+}$, i.e., $\mathrm{Na}_{24}^{2+} \rightarrow \mathrm{Na}_{21}^{+}+\mathrm{Na}_{3}^{+}$, that has been experimentally observed. ${ }^{24}$ As in Ref. 19 we compare the results obtained from selected jellium profiles, defined by the relations between the parameters $\lambda$ and $\sigma$ [Eqs. (4)-(5)] proposed by Blocki and co-workers in the nuclear physics case. ${ }^{20}$ The value of $\Delta$ [Eq. (3)] is fixed by the size of the final fragments (in this case, $\Delta=0.3134$ ).

Figure 2(a) displays the total energy of the system $\mathrm{Na}_{21}^{+}$ $+\mathrm{Na}_{3}^{+}$as a function of fragment separation for two different fission pathways. Both calculations artificially start with a sphere, defined by $\lambda=1-\Delta, \sigma=\Delta$. The dashed line corresponds to the jellium shapes schematically shown at the top of the figure. This parametrization starts with the sphere described before and follows the line corresponding to a cone capped with spheres $\left(\lambda=1-\Delta^{2} / \sigma\right)$ up to an arbitrary value of $\sigma$ where a concave neck starts to form; after that point we assume the fastest variation of the neck $\left[(\lambda-1)^{2}\right.$ $-\sigma^{2}=$ constant $]$ up to $\lambda=0$, and continue with two separated spheres. In the present case, the cluster is forced to elongate up to $s=18.3$ a.u. $(\sigma=1.175)$ and the scission occurs at $s=23.0$ a.u. The energy minimum at $s=11.8$ a.u. is, in fact, the ground state of the parent cluster for this pathway. This particular jellium parametrization is very similar to the shape sequences used in heavy-nuclei fission, ${ }^{20}$ for which the maximum of the fission barrier (saddle point) corresponds to moderate deformations and occurs before scission.

Nevertheless, from the results of dynamics simulations for

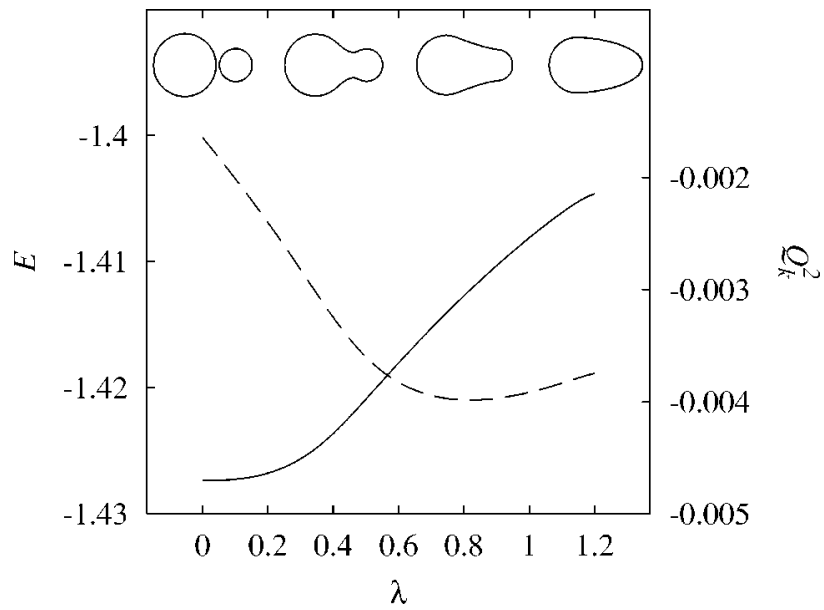

FIG. 3. Energy (solid line) and quadrupole moment in $k$ space (dashed curve) in Hartree atomic units vs the neck deformation parameter $\lambda$ for a fixed value $s=18$ a.u. of the distance between the centers of the emerging fragments for the process $\mathrm{Na}_{24}^{2+} \rightarrow \mathrm{Na}$ ${ }_{21}^{+}+\mathrm{Na}_{3}^{+}$. The jellium profiles for $\lambda=0,0.4,0.8$, and 1.2 are also shown.

small charged metal clusters, ${ }^{25}$ the cluster fission situation seems to be better described by pathways in which the emerging fragments are already formed before reaching the maximum of the barrier. The simplest choice to simulate that situation is the two-sphere parametrization [jellium shapes shown at the bottom of Fig. 2(a), and solid line], defined by $\lambda=1-\sigma$ up to $\sigma=1$ (interpenetrated jellium spheres) and $\lambda=0, \sigma>1$ afterwards (two separated jellium spheres). The scission point of this fission pathway occurs at $s=17.2$ a.u. and corresponds to the ground state of $\mathrm{Na}_{24}^{2+}$, whereas the maximum of the barrier is reached at $s \simeq 22$ a.u.

Starting at the pertinent ground state for each parametrization we have analyzed in Fig. 2(b) the behavior of the quadrupole moment in $k$ space for the fission pathways described in Fig. 2(a). As it is shown the pathway corresponding to the minimum-energy barrier (solid line) shows $Q_{k}^{2}$ closer to zero than the pathway with higher-energy barrier. When the two fragments are well separated one recovers the complete isotropy of the momentum distribution.

On the other hand, we have computed the cluster energy as a function of the parameter $\lambda$ for an arbitrary fixed value of the distance between the emerging fragments (see Fig. 3). This evolution does not correspond to any realistic fission pathway, but also in this case the jellium configuration associated to the minimum-energy value has a nearly vanishing $Q_{k}^{2}$. This result fortifies the conclusion that the most stable configurations have quadrupole moments in $k$ space close to zero, even in fission configurations for which the jellium deformation is fixed a priori.

In summary, we have investigated properties of electronic momentum distribution in spheroidal clusters and fission configurations using the KS formalism. We find that for spheroidal clusters the equilibrium configuration can be determinated by requiring that $Q_{k}^{2}=0$. For fission configurations the quadrupole moment in $k$ space is closer to zero for the pathway that corresponds to the minimum-energy barrier. The main features of the global momentum distribution can be obtained from its monopole component. As in the case of 
spherical clusters, the properties of this monopole component can be understood on the basis of the Slater approach for the local momentum distribution. Deformation can be clearly seen in the single-particle momentum distribution.
The analysis of its shape can be used as an indication of the mixing of various $l$ waves in each deformed orbital.

This work has been supported by DGICYT (Spain), Grant Nos. PB95-0492 and PB95-0123.
${ }^{1}$ W. D. Knight, K. Clemenger, W. A. de Heer, W. A. Saunders, M. Y. Chou, and M. L. Cohen, Phys. Rev. Lett. 52, 2141 (1984).

${ }^{2}$ W. D. Knight, K. Clemenger, W. A. de Heer, and W. A. Saunders, Phys. Rev. B 31, 2539 (1985).

${ }^{3}$ W. A. de Heer, Rev. Mod. Phys. 65, 611 (1993).

${ }^{4}$ M. Brack, Rev. Mod. Phys. 65, 677 (1993), and references therein.

${ }^{5}$ M. M. Kappes, M. Schär, U. Röthlisberger, C. Yeretzian, and E. Schumacher, Chem. Lett. 143, 251 (1988).

${ }^{6}$ C. Bréchignac, Ph. Cahuzac, J. Leygnier, and A. Sarfati, Phys. Rev. Lett. 70, 2036 (1993).

${ }^{7}$ C. Bréchignac, Ph. Cahuzac, F. Carlier, J. Leygneir, and A. Sarfati, Phys. Rev. B 44, 11386 (1991); C. Bréchignac, Ph. Cahuzac, F. Carlier, and M. de Frutos, ibid. 49, 2825 (1994).

${ }^{8}$ A. Bulgac and J. M. Thompson, Phys. Lett. B 383, 127 (1996).

${ }^{9}$ A. Rigo, M. Casas, F. Garcias, E. Moya de Guerra, and P. Sarriguren, Z. Phys. D 40, 294 (1997).

${ }^{10}$ K. Clemenger, Phys. Rev. B 32, 1359 (1985).

${ }^{11}$ S. M. Reimann, M. Brack, and K. Hansen, Z. Phys. D 28, 235 (1993).

${ }^{12}$ W. Ekardt and Z. Penzar, Phys. Rev. B 38, 4273 (1988).

${ }^{13}$ W. Ekardt and Z. Penzar, Phys. Rev. B 43, 1322 (1991).

${ }^{14}$ Z. Penzar and W. Ekardt, Z. Phys. D 17, 69 (1990).

${ }^{15}$ J. Borggreen, P. Chowdhury, N. Kebaili, L. Lündsberg-Nielsen,
K. Lutzenkirchen, M. B. Nielsen, J. Pedersen, and H. D. Rasmussen, Phys. Rev. B 48, 17507 (1993).

${ }^{16}$ P. Meibon, M. Ostergard, J. Borggreen, S. Bjørnholm, and H. D. Rasmussen, Z. Phys. D 40, 258 (1997).

${ }^{17}$ Th. Hirschman, M. Brack, and P.-G. Reinhard, Z. Phys. D 40, 254 (1997).

${ }^{18}$ F. Garcias, A. Mañanes, J. M. López, J. A. Alonso, and M. Barranco, Phys. Rev. B 51, 1897 (1995).

${ }^{19}$ A. Rigo, F. Garcias, J. A. Alonso, J. M. López, M. Barranco, A. Mañanes, and J. Németh, Surf. Rev. Lett. 3, 617 (1996).

${ }^{20}$ J. Blocki, J. Phys. (Paris), Colloq. 45, C6-489 (1984); J. Blocki, R. Planeta, J. Brzychczyk, and K. Grotowski, Z. Phys. A 341, 307 (1992).

${ }^{21}$ J. A. Caballero and E. Moya de Guerra, Nucl. Phys. A 509, 117 (1990).

${ }^{22}$ A. Bohr and B. R. Mottelson, Nuclear Structure (Benjamin, New York, 1975), Vol. II.

${ }^{23}$ E. Moya de Guerra, P. Sarriguren, J. A. Caballero, M. Casas, and D. W. L. Sprung, Nucl. Phys. A 529, 68 (1991).

${ }^{24}$ C. Bréchignac, Ph. Cahuzac, F. Carlier, and M. de Frutos, Phys. Rev. Lett. 64, 2893 (1990).

${ }^{25}$ R. N. Barnett, U. Landman, and G. Rajagopal, Phys. Rev. Lett. 67, 3058 (1991); C. Bréchignac, Ph. Cahuzac, F. Carlier, M. de Frutos, R. N. Barnett, and U. Landman, ibid. 72, 1636 (1994). 